\title{
Enhancing in vivo renal ischemia assessment by high-dynamic-range fluorescence molecular imaging
}

\author{
Yang Gao \\ Yuan Zhou \\ Fei Liu \\ Jianwen Luo
}




\title{
Enhancing in vivo renal ischemia assessment by high-dynamic-range fluorescence molecular imaging
}

\author{
Yang Gao, ${ }^{a}$ Yuan Zhou, ${ }^{a}$ Fei Liu, ${ }^{b, *}$ and Jianwen Luo ${ }^{a, c, *}$ \\ ${ }^{\mathrm{a}}$ Tsinghua University, School of Medicine, Department of Biomedical Engineering, Beijing, China \\ ${ }^{b}$ Beijing Jiaotong University, School of Computer and Information Technology, Beijing, China \\ ${ }^{\mathrm{C} T}$ Tsinghua University, Center for Biomedical Imaging Research, Beijing, China
}

\begin{abstract}
Fluorescence imaging has been used to evaluate the physiological features of renal ischemia in animal model. However, the fluorophore distribution details of the ischemia model could not be fully represented due to the limited dynamic range of the charged-couple device. A high-dynamic-range (HDR) strategy was adopted in renal ischemia fluorescence imaging, both ex vivo and in vivo. The HDR strategy successfully combined ischemia relevant biological features that could only be captured with different exposure times, and then presented these features in the HDR results. The HDR results effectively highlighted the renal ischemic areas with relatively better perfusion and diminished the saturation that resulted from long exposure time. The relative fluorescence intensities of the ischemic kidneys and the image entropy values were significantly higher in the HDR images than in the original images, therefore enhancing the visualization of the renal ischemia model. The results suggest that HDR could serve as a postprocessing strategy to enhance the assessment of in vivo renal ischemia, and HDR fluorescence molecular imaging could be a valuable imaging tool for future studies of clinical ischemia detection and evaluation. ๑ 2018 Society of Photo-Optical Instrumentation Engineers (SPIE) [DOI: 10.1117/1.JBO.23.7.076009]
\end{abstract}

Keywords: fluorescence molecular imaging; high-dynamic-range; fluorophore distribution; renal ischemia assessment.

Paper 180265R received May 7, 2018; accepted for publication Jun. 29, 2018; published online Jul. 18, 2018.

\section{Introduction}

Fluorescence molecular imaging visualizes biological processes at molecular level ${ }^{1-3}$ and provides advantages including nonradioactivity, high detection sensitivity, and relatively low instrumental cost. ${ }^{4,5}$ Low tissue autofluorescence and absorption in the near-infrared (NIR) window benefit the detection performance of fluorescence molecular imaging. ${ }^{6-8}$ Therefore, NIR fluorescence imaging serves as a valuable tool $^{9}$ for research fields including tumor pharmacokinetics, ${ }^{10-12}$ vascular function, ${ }^{13,14}$ and tissue ischemia. ${ }^{15-17}$

Kidneys receive $\sim 20 \%$ of the cardiac output, ${ }^{18}$ and the renal anatomy is vascular-rich. Clinically, renal ischemia could be caused by various reasons, including arterial and venous occlusion, thrombosis, and vasculitis. ${ }^{19,20}$ Renal ischemia often results in serious renal physiology alteration, even leading to severe prognosis. Therefore, in vivo detection and assessment of renal ischemia are important for evaluating renal conditions during ischemia.

For renal physiology studies, previous works have shown that the epidermal growth factor receptor (EGFR) was a valuable target to investigate. ${ }^{21-25}$ An EGFR-binding fluorescent probe named EGF-IRDye was generated by conjugating an NIR dye (IRDye CW800) with the recombinant human EGF protein (which forms specifically binding with EGFR). After entering blood circulation, the EGF-IRDye probe quickly reaches and accumulates in kidneys. In the present study, this probe was

*Address all correspondence to: Jianwen Luo, E-mail: luo_jianwen @ tsinghua .edu.cn; Fei Liu, E-mail: liufei@ bjtu.edu.cn used to indicate renal blood flow characteristics in mouse renal ischemia model.

The limited dynamic range of charged-couple device $(\mathrm{CCD})^{26-32}$ prevents the imaging system from fully capturing the fluorescence intensity difference of imaging targets that have significant different fluorophore concentrations. Consequently, fluorescence images of renal ischemia model using single exposure times could not fully present the ischemia features, due to the significant fluorescence intensity difference between the normal kidney and the ischemic kidney.

Modifying CCD hardware could help to solve this limitation, ${ }^{33-35}$ but hardware modification could be costly and time-consuming. Another strategy adopted multiwavelength approaches including optical spectroscopic imaging ${ }^{17,36-39}$ and autofluorescence imaging ${ }^{40-45}$ to investigate the renal ischemia physiology. However, these approaches have limitations, including altered fluorophore quantum efficiency, ${ }^{39}$ weak or absent correlation between fluorescence and ischemia, ${ }^{41}$ phototoxicity under short-wavelength, ${ }^{43}$ and restricted clinical application. ${ }^{42,43}$

The high-dynamic-range (HDR) method is a widely used method in digital photography that could effectively enhance the dynamic range by combining features of images with different exposures. ${ }^{28,46-48}$ Recently, HDR method has been reported to combine with various imaging modalities and enhance their performance. HDR optical projection tomography (OPT) was developed to resolve details of zebra fish embryos. ${ }^{49}$ HDR laser-scanning microscopy (LSM) was used to facilitate three-dimensional neural segmentation. ${ }^{50}$ HDR fluorescence

$1083-3668 / 2018 / \$ 25.00$ (C) 2018 SPIE 
molecular tomography (FMT) was used to detect tumors with different fluorophore concentrations. ${ }^{51}$ HDR fluorescence laminar optical tomography (FLOT) was used to improve the penetration depth and quantification accuracy of FLOT. ${ }^{52}$

The significant fluorescence intensity differences between the ischemic kidney and normal kidney could reveal physiological information relevant to renal ischemia, but these differences could not be fully presented due to the limited dynamic range. In our work, HDR method has been employed to increase the dynamic range of the fluorescence imaging and it could be a potential strategy for enhancing renal ischemia assessment. To the best of our knowledge, there are no reports of combining HDR with NIR fluorescence imaging to improve renal ischemia assessment.

In this paper, we developed an HDR fluorescence imaging method to enhance the visualization of physiological relevant information of the renal ischemia model in order to improve the assessment of in vivo renal ischemia. First, the CCD response function was recovered. Then, HDR workflow was validated using images of phantoms with different fluorophore concentrations. Last, HDR images of mouse renal ischemia model (both ex vivo and in vivo) were constructed and compared with original images. The results suggested that our approach constitutes an initial step toward enhancing the assessment of in vivo renal ischemia using HDR method and could serve as a postprocessing imaging augmentation strategy for further studies on organ ischemia detection and evaluation.

\section{Methods}

\subsection{Construction of HDR Fluorescence Images}

Our approach for constructing HDR images was developed based on a previously described method. ${ }^{46}$

\subsubsection{Recovering CCD response function}

A series of fluorescence images with multiple exposure times was collected to recover the CCD response function. The image series was selected to ensure that the fluorescence intensity of each image sufficiently overlapped with each other. Twenty four images were selected to recover the response function accurately.

The fluorescence image series consisted of $P$ images, and each image had $N$ pixels. The original fluorescence images with exposure time $t_{j}$ were denoted by $Z_{i j}^{\text {raw }}$, where $i$ is the spatial index over pixels, and $j$ is the image index in the series. The low dynamic range fluorescence images used to calculate CCD response function were denoted as $Z_{i j}$, which were obtained as ${ }^{46}$

$Z_{i j}=Z_{i j}^{\mathrm{raw}}-Z_{i j}^{\mathrm{back}}$

where $Z_{i j}^{\text {back }}$ was the corresponding background fluorescence noise images collected by the imaging platform without any objects.

The scene was assumed static, as the low dynamic range images were collected rapidly that the fluorescence changes could be safely ignored. Therefore, the irradiance $E$ remained constant among images with different exposure times.

The film reciprocity equation could be written as follows: ${ }^{46}$

$Z_{i j}=f\left(E_{i} t_{j}\right)$ where $E_{i}$ was the irradiance at pixel index $i$. Since $f$ was monotonic and invertible, Eq. (2) could be rewritten as ${ }^{46}$

$f^{-1}\left(Z_{i j}\right)=E_{i} t_{i j}$

Taking natural logarithm on both sides of Eq. $(3)^{46}$

$\ln f^{-1}\left(E_{i} t_{j}\right)=\ln E_{i}+\ln t_{j}$.

$\ln f^{-1}$ was redefined as $g=\ln f^{-1}$ to simplify notation ${ }^{46}$

$g\left(Z_{i j}\right)=\ln E_{i}+\ln t_{j}$

The fluorescence intensity values of $Z_{i j}$ were finite, therefore recovering $g\left(Z_{i j}\right)$ only required finite values. The least and the greatest pixel values of $Z_{i j}$ were denoted by $Z_{\min }$ and $Z_{\max }$, respectively. Therefore, $g\left(Z_{i j}\right)$ could be recovered by finding $Z_{\max }-Z_{\min }+1$ values of $g\left(Z_{i j}\right)$ and $N$ values of $\ln E_{i}$ that minimized the following quadratic objective function: ${ }^{46}$

$$
\begin{aligned}
\mathrm{O}= & \sum_{i=1}^{N} \sum_{j=1}^{P}\left\{\omega(z)\left[g\left(Z_{i j}\right)-\ln E_{i}-\ln t_{j}\right]\right\}^{2} \\
& +\lambda \sum_{z=Z_{\min }+1}^{Z_{\max }-1}\left[\omega(z) g^{\prime \prime}(z)\right]^{2},
\end{aligned}
$$

where $\lambda$ was an empirically determined smoothing scalar (i.e., regularization parameter), $\omega(z)$ was a weighting function to emphasize the middle portion of the response curve ${ }^{46}$

$w(z)=\left\{\begin{array}{ll}z-Z_{\text {min }}, & z<Z_{\text {mid }} \\ Z_{\max }-z, & z<Z_{\text {mid }}\end{array}\right.$,

the medium fluorescence intensity between $Z_{\min }$ and $Z_{\max }$ was denoted by $Z_{\text {mid }}=\frac{1}{2}\left(Z_{\min }+Z_{\max }\right)$, and $g^{\prime \prime}(z)$ was the second derivative of $g(z)$, numerically calculated as ${ }^{46}$

$g^{\prime \prime}(z)=g(z-1)-2 g(z)+g(z+1)$.

In order to properly locate the CCD response curve, another constraint was introduced to ensure the pixel with $Z_{\text {mid }}$ have the unit exposure, as follows: ${ }^{46}$

$g\left(Z_{\text {mid }}\right)=0$.

Not all available pixels were needed for solving Eq. (6). In order to keep Eq. (6) as an overdetermined equation, the following condition should be met: ${ }^{46}$

$N(P-1)>Z_{\max }-Z_{\min }$.

In our dataset, the fluorescence intensity range $Z_{\max }-Z_{\min }=16,032$ and the number of fluorescence images $P=24$. Therefore at least 698 pixels should be sampled from each image $(N>697)$. In the actual calculation process, 1200 pixels were sampled from each image and used for calculating the CCD response curve. These pixels were chosen from regions with low intensity variation, and they distributed evenly from $Z_{\min }$ to $Z_{\max }$.

Minimizing Eq. (6) was a linear least squares problem, and it was solved using the singular value decomposition method. ${ }^{46,53}$ Then, the response curve was fitted as follows: 
$f(x)=a \exp \left\{-[(x-b) / c]^{2}\right\}$,

and then plotted along with original data points.

\subsubsection{Constructing HDR fluorescence images}

HDR fluorescence images were constructed as follows: ${ }^{51}$

$H_{\log }=\frac{\sum_{i=1}^{P} \omega\left(Z_{i j}\right)\left[g\left(Z_{i j}\right)-\ln t_{j}\right]}{\sum_{i=1}^{P} \omega\left(Z_{i j}\right)}$.

The logarithmic form of HDR fluorescence image $\left(H_{\log }\right)$ was then mapped to pixel values $H$ through the tone-mapping process ${ }^{53,54}$ to properly display the information contained in the HDR images.

\subsection{Experimental Design}

\subsubsection{Phantom study: recovering $C C D$ response function}

The fluorescent probe was EGF-conjugated IRDye 800CW (EGF-IRDye; LI-COR Biosciences, Lincoln, Nebraska). A homemade reflectance fluorescence imaging system $^{12,15,16,55}$ was used in this work for fluorescence molecular imaging (Fig. 1). A 300-W xeon lamp (MAX-302, Asahi Spectra, Torrance, California) was used as the excitation source. The excitation light was filtered through a $770 \pm 6 \mathrm{~nm}$ band-pass filter (XBPA770, Asahi Spectra, Torrance, California) before reaching the object at a power density of $0.03 \mathrm{~mW} / \mathrm{cm}^{2}$. The emitted fluorescence was filtered through an $800 \pm 10 \mathrm{~nm}$ band-pass filter (FBH800-10 Premium Bandpass Filter, Thorlabs, Newton, New Jersey) and then detected by a $512 \times 512$ pixel, $-70^{\circ} \mathrm{C}$ electron multiplying CCD (EMCCD) camera (iXon DU-897, Andor Technologies, Belfast, Northern Ireland, United Kingdom) coupled with a $35-\mathrm{mm} f / 1.6$ lens (C3514-M, Pentax, Tokyo, Japan). Each pixel has a size of $0.16 \times 0.16 \mathrm{~mm}^{2}$.

Fluorescence images of a phantom with 24 different exposure times (i.e., 0.01, 0.02, 0.05, 0.10, 0.20, 0.25, 0.30, 0.40, $0.50,0.60,0.70,0.80,0.90,1.00,1.10,1.20,1.301 .40,1.50$,

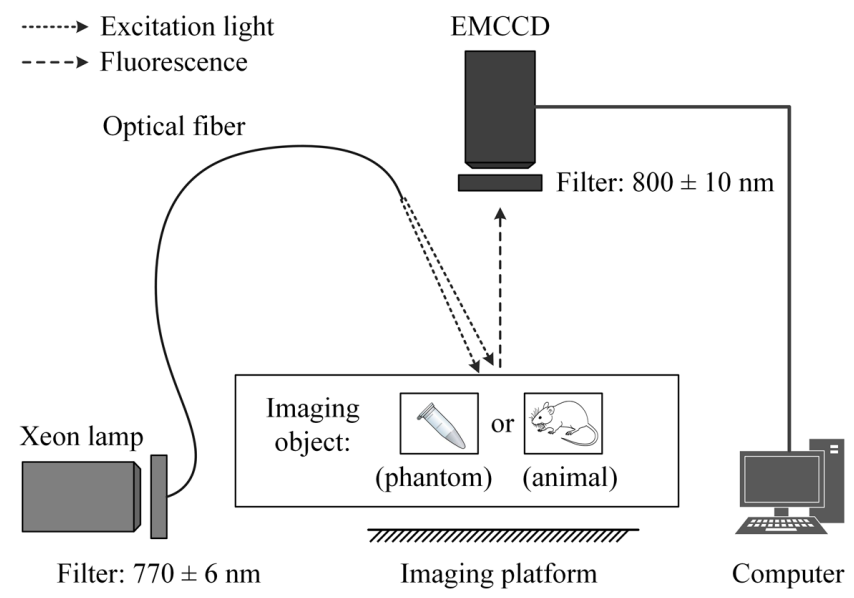

Fig. 1 A schematic of the fluorescence molecular imaging system. The excitation light from the xeon lamp is filtered through a $770 \pm 6 \mathrm{~nm}$ filter, and the emitted fluorescence from the imaging object (phantom or animal) is filtered through an $800 \pm 10 \mathrm{~nm}$ filter before reaching the EMCCD.
$1.601 .701 .80,1.90$, and $2.00 \mathrm{~s}$ ) were collected to recover the CCD response function. The phantom was a $0.5-\mathrm{ml}$ centrifugal tube (Eppendorf, Hamburg, Germany) filled with $0.2 \mathrm{ml}$ $6.0 \mathrm{nmol} / \mathrm{ml}$ EGF-IRDye dissolved in $1 \times$ phosphate buffer saline (PBS) (HyClone, Logan, Utah). This image dataset was collected and the fluorophore concentration of phantom remained consistent throughout the whole collection process.

The excitation light was adjusted to ensure that it was focused at the center of the FOV, and then the phantom was placed at the center of the FOV to ensure that the emission fluorescence was captured on the center of the CCD. Note that the source-to-target distance was $\sim 28 \mathrm{~cm}$ and remained unchanged throughout the experiments.

\subsubsection{Phantom study: validating HDR workflow}

Phantoms with different concentrations of EGF-IRDye solution were used to validate the HDR workflow. Three 0.5 -ml centrifugal tubes were filled with $1.2,3.6$, and $6.0 \mathrm{nmol} / \mathrm{ml} \mathrm{EGF-}$ IRDye solution and denoted as P1, P2, and P3, respectively $(0.2 \mathrm{ml}$ in each tube). Fluorescence images were collected with exposure time of $0.8,1.8$, and $2.6 \mathrm{~s}$. Then these fluorescence images were used to construct the HDR result image of the phantoms using Eq. (12).

\subsubsection{Animal studies: imaging ex vivo and in vivo renal ischemia}

8-week-old female BALB/c-nude mice (each weighted 20 to $22 \mathrm{~g}$ ) were placed under anesthesia using $2.5 \%$ isofluraneoxygen mixture. A dorsal laparotomy was conducted. Then, the arterial and venous flow of the left kidney (in dorsal view) was simultaneously occluded using cotton thread, resulting in unilateral renal ischemia. The right kidney was not ligated, to serve as the normal control. Animal experiments were conducted with approval from the Ethics Committee of Tsinghua University.

After the renal ischemia model was established, each mouse was intravenously injected a bolus of 1-nmol EGF-IRDye dissolved in $0.3 \mathrm{ml} 1 \times$ PBS. The dose of EGF-IRDye injected per body weight ranged from 0.045 to $0.050 \mathrm{nmol} / \mathrm{g}$, approximately. Then, the mice were divided into two groups randomly (three mice per group). One group was used for in vivo imaging while the kidneys of the other group were harvested for ex vivo imaging.

Fluorescence images were collected $60 \mathrm{~min}$ after renal ischemia was induced. The positions of the kidneys (ex vivo study) and mouse (in vivo study) were maintained through the entire imaging process. The exposure times used in the animal studies were $3.2,4.0$, and $4.4 \mathrm{~s}$ for ex vivo imaging, and 3.6, 4.2, and $4.6 \mathrm{~s}$ for in vivo imaging. The whole imaging process normally takes $<1$ min. In the in vivo imaging, tissue surrounding kidneys was covered with black cloth to diminish the fluorescence from outside the renal ischemia model. HDR fluorescence images were constructed as described in Sec. 2.1.

\subsection{Evaluation Metrics}

\subsubsection{Relative fluorescence intensity of the phantom}

The fluorescence intensity of a phantom $\left(\mathrm{FI}_{\text {phantom }}\right)$ was the average of fluorescence intensities of $105 \times 5$ rectangular region-ofinterest (ROI) in 10 positions of the phantom. Then, the relative fluorescence intensity $r_{\text {phantom }}$ was calculated by normalizing 
$\mathrm{FI}_{\text {phantom }}$ using upper fluorescence intensity limit of the CCD $\left(\mathrm{FI}_{\max }\right)$

$r_{\text {phantom }}=\frac{\mathrm{FI}_{\text {phantom }}}{\mathrm{FI}_{\max }}$,

where $\mathrm{FI}_{\max }=16,383$. The relative intensity was used for conveniently indicating the portion of the fluorescence intensity relative to the maximum. Then for each phantom, the relative fluorescence intensity was plotted against its fluorescent probe concentration.

\subsubsection{Relative fluorescence intensity of the ischemic kidney}

In this study, the relative fluorescence intensity of the ischemic kidney $\left(r_{\text {ischemic kidney }}\right)$ is defined as the ratio between the fluorescence intensity of the ischemic kidneys and that of the control kidneys, as follows:

$r_{\text {ischemic kidney }}=\frac{\mathrm{FI}_{\text {ischemic }}}{\mathrm{FI}_{\text {control }}}$,

where $\mathrm{FI}_{\text {ischemic }}$ and $\mathrm{FI}_{\text {control }}$ are the average fluorescence intensities of the ROI surrounding the pixel with the maximum fluorescence intensity in the ischemic kidneys and control kidneys, respectively. This relative fluorescence intensity is used for comparing the relative fluorescence intensity of the ischemic kidneys and control kidneys.

\subsubsection{Image entropy}

The amount of visual information supplied by the original and HDR images were quantitatively measured by a metric called image entropy $(E),{ }^{56,57}$ which is defined as follows:

$E=-\sum_{k=0}^{M-1} p_{k} \log _{2}\left(p_{k}\right)$,

(a)

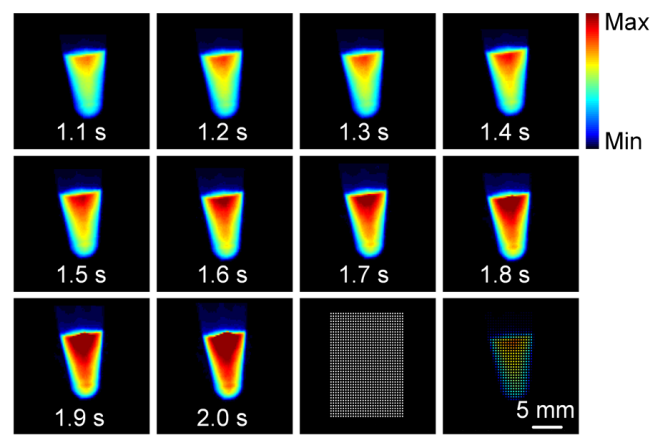

where $M$ is the number of intensity levels in the image and $p_{k}$ is the probability associated with intensity level $k$.

\subsubsection{Statistical analysis}

Data were expressed as mean \pm standard deviation. Statistical differences were calculated by one-tailed Student's $t$ test. A $p$-value $<0.05$ was considered statistically significant. Mean, standard deviation, and $p$-value were calculated using Excel 2016 (Microsoft Corp., Seattle, Washington).

\section{Results}

\subsection{Recovered CCD Response Curve from Multiexposure Fluorescence Images}

Fluorescence images of a phantom are collected with different exposure times [Fig. 2(a)]. The sampling positions and a representative fluorescence image after sampling are shown [bottom right, Fig. 2(a)]. The original data points are represented by red dots, whereas the fitted response curve is plotted as black line [Fig. 2(b)].

\subsection{Validation of HDR Workflow}

Phantoms P1, P2, and P3 are imaged with a series of increasing exposure times [Figs. 3(a)-3(c)]. The HDR fluorescence image constructed according to Sec. 2.1 was shown in Fig. 3(d).

With the exposure time as $0.8 \mathrm{~s}$, fluorescence intensity of P1 is close to the background level, although P2 and P3 can be clearly visualized [Fig. 3(a)]. In order to sufficiently display the details of $\mathrm{P} 1$, the exposure time has to be extended. As the exposure time is increased to $1.8 \mathrm{~s}, \mathrm{P} 1$ becomes distinguishable, but some pixels in $\mathrm{P} 3$ begin to reach saturation level [indicated by the white arrow in Fig. 3(b)]. When the exposure time is further extended to $2.6 \mathrm{~s}$, the top part of P1 with peak intensity finally can be distinguished. However, both P2 and P3 suffer from saturation and distortion [white arrows, Fig. 3(c)], due to the increased exposure time.

In the HDR result, the fluorescence intensity of $\mathrm{P} 1$ is further increased compared to that of P1 in Fig. 3(c), and the area with

(b)

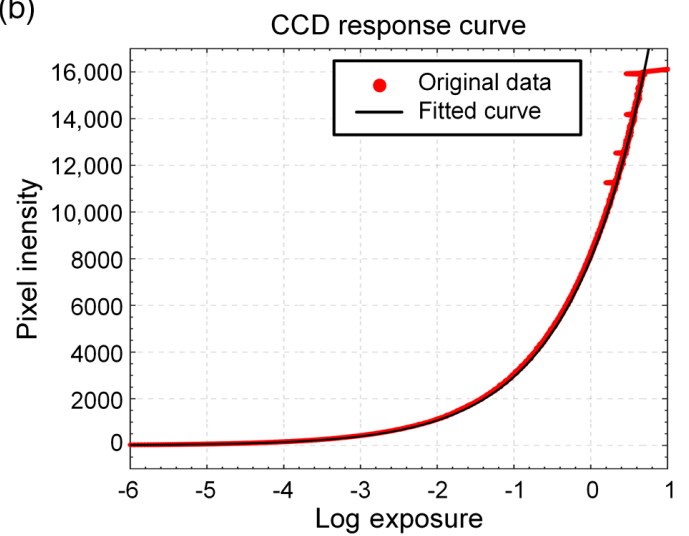

Fig. 2 CCD response curve calculated using fluorescence images collected with different exposure times. (a) Twenty four fluorescence images of a phantom filled with fluorescent probe EGF-IRDye were taken with increasing exposure times (10 representative images were shown). 1200 points $(40 \times 30)$ are sampled from each image to calculate the CCD response curve, and the locations of sampling points are illustrated (bottom row, second from right). A representative fluorescence image after sampling is shown (right bottom corner). (b) CCD response curve. The original data of log exposure and pixel intensity are represented in red, and the fitted response curve is shown in black. 

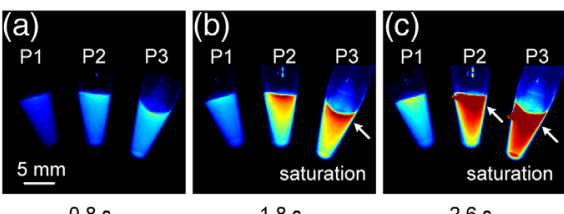

$2.6 \mathrm{~s}$
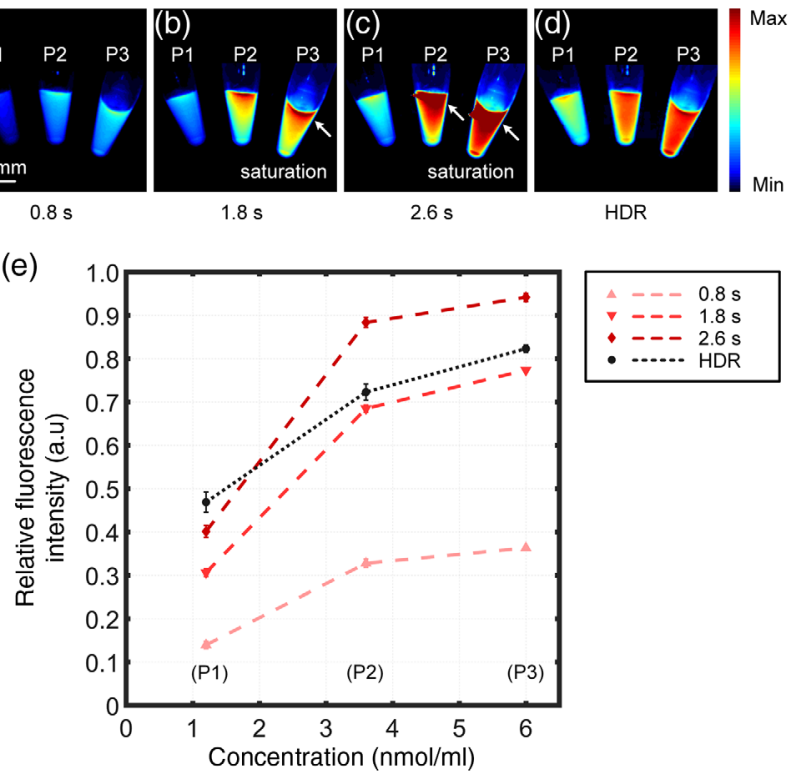

Fig. 3 (a)-(c) Original fluorescence images of three phantoms (labeled as P1, P2, and P3) filled with 1.2, 3.6, and $6.0 \mathrm{nmol} / \mathrm{ml}$ EGF-IRDye probe, with the exposure times of $0.8,1.8$, and $2.6 \mathrm{~s}$, respectively. Saturation areas are indicated by white arrows. (d) HDR result of the phantoms. (e) Relative fluorescence intensity values of phantoms of different concentrations. The error bars indicate the standard deviations of the results from three experiments.

peak fluorescence intensity remains visible. Moreover, the saturation of P2 and P3 is diminished. Relative fluorescence intensity values of these three phantoms are extracted as described in Sec. 2 and plotted against the probe concentration [Fig. 3(e)].
The HDR result decreases the relative fluorescence intensity of the phantoms that appear saturated in Fig. 3(c), thus suppresses the saturation. The HDR result also increases the intensity of P1 compared to its original relative intensity even with the longest exposure time. Moreover, the HDR result shows a better linear relationship between the relative fluorescence intensity and probe concentration.

\subsection{HDR Results of the Mouse Renal Ischemia Model}

\subsubsection{Ex vivo HDR results}

Ischemic and control kidneys are harvested and imaged ex vivo (Fig. 4). In the renal ischemia model, the blood vessels were not completely occluded; therefore, the blood stream was partially remained, resulting in the renal area with better perfusion. The ischemic kidneys are shown on the right side, whereas the control kidneys are on the left. The positions of kidneys are labeled ( $\mathrm{H}=$ head; $\mathrm{T}=$ tail; $\mathrm{L}=$ left; $\mathrm{R}=$ right $)$. With the exposure time as $3.2 \mathrm{~s}$, the ischemic kidney exhibits low fluorescence intensity, and the control kidney is not sufficiently visualized [Fig. 4(a)]. Increasing the exposure time to $4.0 \mathrm{~s}$ increases the overall fluorescence intensity of the ischemic kidney, but the renal area that has better perfusion in the ischemic kidney could not be clearly distinguished [Fig. 4(b)]. When the exposure time is increased to $4.4 \mathrm{~s}$, the relatively better perfused renal area starts to distinguish itself from the rest of the kidney, as the overall fluorescence intensity of the ischemic kidney is further increased. However, large portion of the normal kidney is saturated [the white arrow, Fig. 4(c)].

In the HDR result of the ex vivo study [Fig. 4(d)], the fluorescence intensity of the ischemic kidney is further increased, compared to that of the ischemic kidney in original image

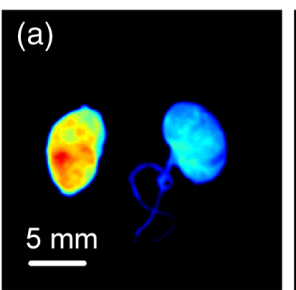

$3.2 \mathrm{~s}$

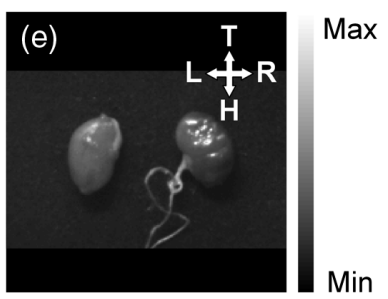

Bright-field

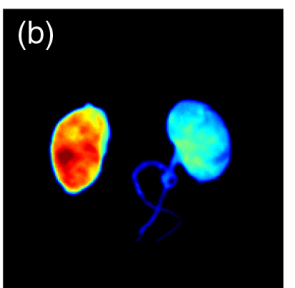

$4.0 \mathrm{~s}$

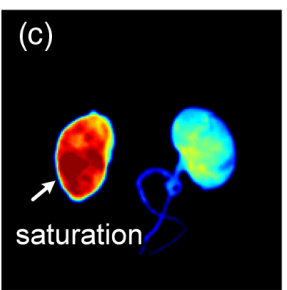

$4.4 \mathrm{~s}$

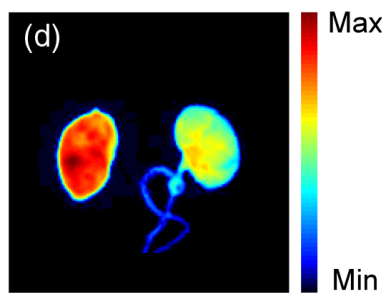

HDR

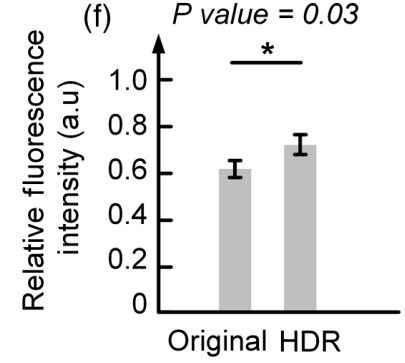

$(4.4 \mathrm{~s})$

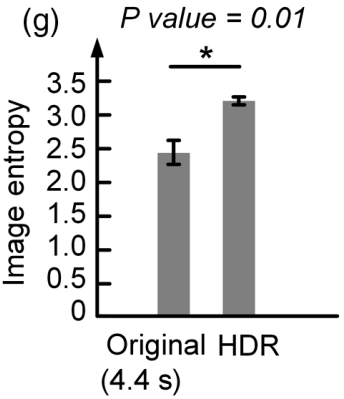

Fig. 4 HDR results of ex vivo imaging of renal ischemia model. (a)-(c) Original fluorescence images of ex vivo mouse renal ischemia model, taken with exposure times of $3.2,4.0$, and $4.4 \mathrm{~s}$, respectively. Control kidneys are on the left while ischemia kidneys are on the right. Saturation areas are indicated by white arrows. (d) HDR image of the ex vivo ischemia model. (e) Bright-field image. The orientation of kidney is labeled $(\mathrm{H}$, head; $\mathrm{T}$, tail; $\mathrm{L}$, left; $\mathrm{R}$, right). The HDR image is compared with the original fluorescence image (with exposure time of $4.4 \mathrm{~s}$ ) in terms of $(\mathrm{f})$ the relative fluorescence intensity of the ischemic kidneys, and $(\mathrm{g})$ the image entropy values, respectively $(n=3)$. The error bars indicate the standard deviations. 
with the longest exposure time [Fig. 4(c)]. The area with better perfusion in the ischemic kidney becomes clearly distinguishable. Moreover, the saturation in control kidney is largely reduced. The area close to the center of the normal kidney remains relatively high fluorescence intensity in the HDR result. Then, the HDR result is compared to the original image that has the longest exposure time ( $4.4 \mathrm{~s})$. The HDR result shows higher relative fluorescence intensity of the ischemic kidneys $(0.68$, 0.75 , and 0.72 for the three mice, respectively) than the original image $(0.58,0.64$, and 0.60$)$ [Fig. 4(f)], as well as higher image entropy values $(3.20,3.26$, and 3.25 , respectively) than the original image $(2.45,2.70$, and 2.27) [Fig. 4(g)].

\subsubsection{In vivo HDR results}

The mouse renal ischemia model was also imaged in vivo (Fig. 5). Mouse body position is labeled ( $\mathrm{H}=$ head; $\mathrm{T}=$ tail; $\mathrm{L}=$ left; $\mathrm{R}=$ right). When the exposure time is $3.6 \mathrm{~s}$, fluorescence intensity of the ischemic kidney is close to the background level, leaving the fluorescence distribution pattern difficult to detect [Fig. 5(a)]. As the exposure time increases to $4.2 \mathrm{~s}$, the overall fluorescence intensity of the ischemic kidney is elevated, but the relatively better perfused area remains unclear [Fig. 5(b)]. With the exposure time further extends to $4.6 \mathrm{~s}$ [Fig. 5(c)], the better perfused area in the ischemic kidney begins to distinguish itself. However, large part of the control kidney is saturated (indicated by the white arrow), which is similar to the saturation in Fig. 4(c).

In the HDR result of the in vivo study [Fig. 5(d)], the fluorescence intensity of the ischemic kidney is increased. Wedgeshaped areas with better blood perfusion in the ischemic kidney could be clearly visualized, suggesting that the HDR result preserves more physiological characteristics than the original images. Moreover, saturation in the control kidney is reduced, and the center area remains to have peak fluorescence intensity. The HDR result is compared to the original image taken with the longest exposure time (4.6 s). The HDR result exhibits higher relative fluorescence intensity of the ischemic kidneys $(0.72$, 0.73 , and 0.72 , respectively) compared to the original image (0.61, 0.63, and 0.59) [Fig. 5(f)] and also shows higher image entropy values $(2.92,3.05$, and 2.83 , respectively) than the original image $(2.35,2.47$, and 2.34) [Fig. 5(g)].

\section{Discussion}

The main goal of this study was to enhance the in vivo assessment of renal ischemia by incorporating HDR method with fluorescence imaging. HDR images of both phantoms and mouse renal ischemia model were constructed based on the original fluorescence images taken with multiple exposure time. The results suggested that the HDR preserved physiological characteristics of both ischemic and control kidneys by highlighting the fluorophore distribution details and diminishing saturation caused by limited dynamic range.

Previous works on enhancing renal ischemia assessment include spectroscopic imaging ${ }^{17,36-39}$ and autofluorescence imaging. ${ }^{40-45}$ These approaches have improved the assessment of ischemic physiology; however, they have limitations such as interference with imaging process due to hypothermic preservation, ${ }^{36}$ altered fluorophore quantum efficiency, ${ }^{39}$ phototoxicity, ${ }^{43}$ and limited application in clinical settings. ${ }^{42,43}$

The fluorescence intensity values and the scene exposure have a nonlinear relationship [Fig. 2(b)], due to the nonlinear mapping of $\mathrm{CCD} .{ }^{46}$ Consequently, a suitable exposure time has to be determined according to the fluorescence intensity

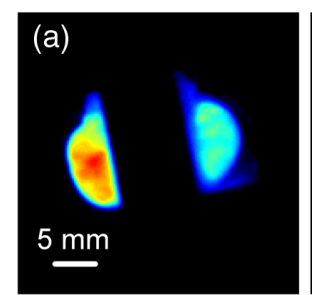

$3.6 \mathrm{~s}$

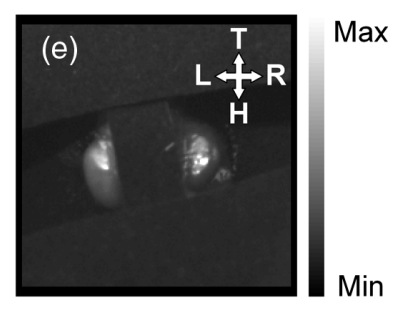

Bright-field

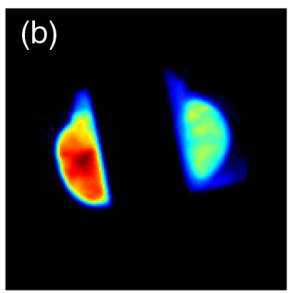

$4.2 \mathrm{~s}$

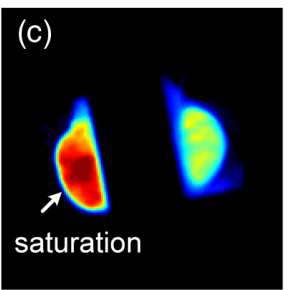

$4.6 \mathrm{~s}$

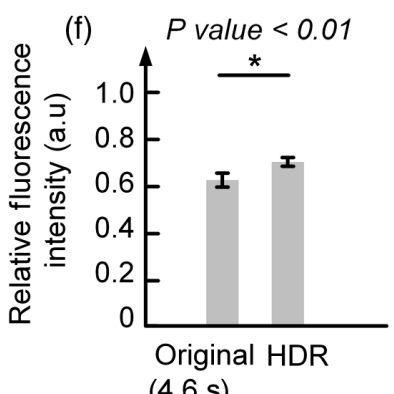

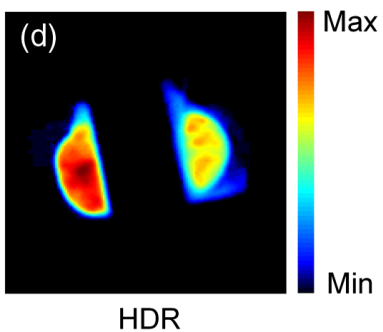

(g) $\quad P$ value $<0.01$

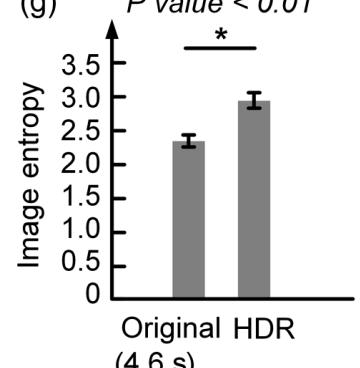

Fig. 5 HDR results of in vivo imaging of renal ischemia model. (a)-(c) Original fluorescence images of in vivo mouse renal ischemia model, with exposure times of 3.6, 4.2, and $4.6 \mathrm{~s}$, respectively. Control kidneys are on the left while ischemic kidneys are on the right. Saturation areas are indicated by white arrows. (d) HDR image of the in vivo ischemia model. Note that in the HDR image, characteristics of fluorescence distribution within the ischemia kidney are presented, and saturation in the control kidney area is diminished. (e) Bright-field image. Mouse position is labeled ( $H$, head; T, tail; L, left; R, right). The HDR image is compared with the original fluorescence image (with exposure time of $4.6 \mathrm{~s}$ ) in terms of (f) the relative fluorescence intensity of the ischemic kidneys and $(\mathrm{g})$ the image entropy values, respectively $(n=3)$. The error bars indicate the standard deviations. 
of the imaging targets in order to avoid saturation. However, if imaging targets in the FOV have large fluorescence intensity differences, it would be difficult to find a proper exposure time to ensure both preserving the details of the low intensity targets and avoiding saturation in the high intensity targets, because the dynamic range of CCD is limited.

This limitation could be improved by the HDR method. HDR has been integrated with several imaging modalities, including OPT,${ }^{49}$ LSM $^{50}{ }^{\text {FMT }},{ }^{51}$ and FLOT ${ }^{52}$ In these studies, HDR has helped to resolve details of imaging targets ${ }^{49,51}$ and to enhance the detection capability of the imaging apparatus. ${ }^{50,52}$

We have adopted a similar strategy. In our work, fluorescence images with different exposure times were mathematically merged by HDR method ${ }^{46,54}$ to preserve physiological features of targets with large intensity differences and enhance in vivo renal ischemia assessment. It is convenient to collect fluorescence images using different exposure times, because the animal position is fixed during the imaging process. Note that exposure times used in phantom studies were different from those in animal studies because of overall fluorescence intensity differences between these two conditions.

The HDR results of phantom studies effectively reduced the saturation, and further increased the fluorescence level of low intensity targets, maintaining a better linearly relationship between probe concentration and fluorescence intensity [Figs. 3(d)-3(e)]. This showed that HDR method has the potential to overcome dynamic range limitation and display imaging targets more accurately.

In the animal studies, ischemic kidneys and control kidneys had significantly different fluorescence intensity level, but this difference was not fully presented with single exposure time. The HDR results of animal studies suggested that our approach could resolve this issue and enhance assessment of renal ischemia from two aspects. The first one is improving the visualization of ischemic kidneys. The HDR results showed significantly higher relative fluorescence intensity of ischemic kidneys, compared to even the original images with the longest exposure times, both ex vivo [Fig. 4(d) vs. Fig. 4(c)] and in vivo [Fig. 5(d) vs. Fig. 5(c)]. This could be validated by the comparison of relative fluorescence intensity of ischemic kidneys illustrated in Figs. 4(f) and 5(f). In addition, HDR results showed more details of the area with better perfusion in the ischemic kidneys. This could be validated by the increased image entropy values shown in Figs. 4(g) and 5(g). The second aspect is suppressing saturation in control kidneys. Saturation became more severe as the exposure time increased and eventually covered major parts of the control kidneys [Figs. 4(c) and 5(c)]. The HDR results suppressed the saturation and maintained the fluorophore distribution pattern in the control kidneys [Fig. 4(d) and $5(\mathrm{~d})]$.

A number of limitations might have influenced the results. First, the animal ischemia model was not strictly static because of animal respiration and renal blood circulation. This might introduce slight disturbance to the results. Second, the exposure times for three types of images required by HDR (insufficiently exposed, normally exposed and overexposed) were selected empirically. For instance, to reach overexposure, 2.6 seconds was chosen for phantom study while 4.6 seconds was chosen for the in vivo ischemia model because of their difference in fluorescence intensity level. Third, the ischemia status is limited. In addition, the gap between the vascular supply profiles and the cellular mechanisms for renal ischemia needs to be explored.
Furthermore, our method needs to be validated using a larger cohort size.

Future work will focus on accelerating data collection to reduce potential disturbance from animal movement. We also intend to investigate the appropriate selection of exposure times based on the overall fluorescence intensity level. More ischemia models, especially the models that closely resemble the clinical practice, need to be established with different occlusion times and used for further evaluating the HDR fluorescence imaging approach. Finally yet importantly, we need to investigate the underlying cellular mechanisms of renal ischemia based on previous studies,${ }^{58-61}$ by tracking the concentration and distribution of key molecules in the ischemia mechanisms using specific fluorescent probes, for expanding our understanding of the renal ischemia progress.

The results of our study indicate that the visualization of renal ischemia model could be effectively improved by the HDR-based imaging approach. The HDR results successfully combined features related to renal ischemia that could only be captured with very different exposure times into a single image. HDR method has the potential for contributing to the medical practice, including providing real-time intraoperative imaging results, removing imaging disturbance due to surgical instruments, helping physicians to interpret the biopsy results and improve the efficacy of other modern imaging modalities. This approach also has the potential for providing more quantitative fluorescence estimation, improving clinical renal ischemia detection and assessment, and could serve as a valuable imaging tool for organ function evaluation.

\section{Disclosures}

The authors have no competing financial interests to disclose in this manuscript.

\section{Acknowledgments}

This work was supported by the National Key R\&D Program of China (Grant No. 2016YFC0102200), the National Natural Science Foundation of China (Grant Nos. 81471665 and 81561168023), and the Fundamental Research Funds for the Central Universities under Grant No. 2017RC023. We would like to thank Wei Liu from the Department of Biomedical Engineering at School of Medicine, Tsinghua University, for his help with the animal experiments.

\section{References}

1. R. Weissleder and U. Mahmood, "Molecular imaging," Radiology 219(2), 316-333 (2001).

2. R. Weissleder, "Molecular imaging in cancer," Science 312(5777), 1168-1171 (2006).

3. M. Rudin and R. Weissleder, "Molecular imaging in drug discovery and development," Nat. Rev. Drug Discov. 2(2), 123-131 (2003).

4. F. Liu et al., "Monitoring of tumor response to cisplatin with simultaneous fluorescence and positron emission tomography: a feasibility study," J. Biophotonics 7(11-12), 889-896 (2014).

5. X. He, K. Wang, and Z. Cheng, "In vivo near-infrared fluorescence imaging of cancer with nanoparticle-based probes," Wiley Interdiscip. Rev. Nanomed. Nanotechnol. 2(4), 349-366 (2010).

6. J. V. Frangioni, "In vivo near-infrared fluorescence imaging," Curr. Opin. Chem. Biol. 7(5), 626-634 (2003).

7. V. Ntziachristos, C. Bremer, and R. Weissleder, "Fluorescence imaging with near-infrared light: new technological advances that enable in vivo molecular imaging," Eur. Radiol. 13(1), 195-208 (2003). 
8. S. A. Hilderbrand and R. Weissleder, "Near-infrared fluorescence: application to in vivo molecular imaging," Curr. Opin. Chem. Biol. 14(1), 71-79 (2010)

9. E. M. Sevick-Muraca, "Translation of near-infrared fluorescence imaging technologies: emerging clinical applications," Annu. Rev. Med. 63(1), 217-231 (2012).

10. G. L. Zhang et al., "A direct method with structural priors for imaging pharmacokinetic parameters in dynamic fluorescence molecular tomography," IEEE Trans. Biomed. Eng. 61(3), 986-990 (2014).

11. N. Huang et al., "Efficacy of NGR peptide-modified PEGylated quantum dots for crossing the blood-brain barrier and targeted fluorescence imaging of glioma and tumor vasculature," Nanomed. Nanotechnol. Biol. Med. 13(1), 83-93 (2017).

12. Y. Gao et al., "Facilitating in vivo tumor localization by principal component analysis based on dynamic fluorescence molecular imaging," J. Biomed. Opt. 22(9), 1-9 (2017).

13. G. Hong et al., "Multifunctional in vivo vascular imaging using nearinfrared II fluorescence," Nat. Med. 18(1), 1841-1846 (2012).

14. H. N. D. Le et al., "Through-skull vasculature assessment using fluorescence brain imaging on murine models at around $800 \mathrm{~nm}$," Proc. SPIE 10051, 100510M (2017).

15. W. Cai et al., "Effects of temperature on multiparametric evaluation of hindlimb ischemia with dynamic fluorescence imaging," J. Biophotonics 10(6-7), 811-820 (2017).

16. H. Guang et al., "Multiparametric evaluation of hindlimb ischemia using time-series indocyanine green fluorescence imaging," J. Biophotonics 10(3), 456-464 (2017).

17. R. N. Raman et al., "Predictive assessment of kidney functional recovery following ischemic injury using optical spectroscopy," J. Biomed. Opt. 22(5), 056001 (2017).

18. D. C. Eaton and J. P. Pooler, Vander's Renal Physiology, 8th ed., Lange Medical Books/McGraw-Hill, New York (2004).

19. Z. J. Ricci et al., "Solid organ abdominal ischemia, part I: clinical features, etiology, imaging findings, and management," Clin. Imaging 40(4), 720-731 (2016).

20. A. Kawashima et al., "CT evaluation of renovascular disease," RadioGraphics 20(5), 1321-1340 (2000).

21. J. Chen, J.-K. Chen, and R. C. Harris, "Deletion of the epidermal growth factor receptor in renal proximal tubule epithelial cells delays recovery from acute kidney injury," Kidney Int. 82(1), 45-52 (2012).

22. J. Tang, N. Liu, and S. Zhuang, "Role of epidermal growth factor receptor in acute and chronic kidney injury," Kidney Int. 83(5), 804-810 (2013).

23. S. He et al., "EGFR activity is required for renal tubular cell dedifferentiation and proliferation in a murine model of folic acid-induced acute kidney injury," Am. J. Physiol. Renal Physiol. 304(4), F356-F366 (2013).

24. J. A. Smith, L. J. Stallons, and R. G. Schnellmann, "Renal cortical hexokinase and pentose phosphate pathway activation through the EGFR/ Akt signaling pathway in endotoxin-induced acute kidney injury," Am. J. Physiol. Renal Physiol. 307(4), F435-F444 (2014).

25. H. M. Kok et al., "Targeting CTGF, EGF and PDGF pathways to prevent progression of kidney disease," Nat. Rev. Nephrol. 10(1), 700-711 (2014).

26. M. A. Robertson, S. Borman, and R. L. Stevenson, "Dynamic range improvement through multiple exposures," in Proc. of Int. Conf. on Image Processing (Cat. 99CH36348), Vol. 153, pp. 159-163 (1999).

27. O. Yadid-Pecht, "Wide-dynamic-range sensors," Opt. Eng. 38(10), 1650-1660 (1999).

28. M. A. Robertson, S. Borman, and R. L. Stevenson, "Estimationtheoretic approach to dynamic range enhancement using multiple exposures," J. Electron. Imaging 12(2), 219-228 (2003).

29. M. N. Inanici, "Evaluation of high dynamic range photography as a luminance data acquisition system," Light. Res. Technol. 38(2), 123-134 (2006).

30. G. Reinaudi et al., "Strong saturation absorption imaging of dense clouds of ultracold atoms," Opt. Lett. 32(21), 3143-3145 (2007).

31. H. Ding et al., "Fourier transform light scattering of inhomogeneous and dynamic structures," Phys. Rev. Lett. 101(23), 238102 (2008).

32. A. Srikantha and D. Sidibe, "Ghost detection and removal for high dynamic range images: recent advances," Signal Process. Image Commun. 27(6), 650-662 (2012).
33. B. Burkey, W. C. Chang, and T. H. Lee, "Reducing Dark Current in Charge Coupled Devices," Eastman Kodak Company, Rochester, New York (1992).

34. R. Widenhorn et al., "Temperature dependence of dark current in a CCD," Proc. SPIE 4669, 193-201 (2002).

35. D. L. Losee and C. Parks, "Method for Reducing Dark Current," Eastman Kodak Company, Rochester, New York (2006).

36. A. P. Michalopoulou et al., "Spectroscopic imaging for detection of ischemic injury in rat kidneys by use of changes in intrinsic optical properties," Appl. Opt. 44(11), 2024-2032 (2005).

37. M. F. Cassini et al., "Fluorescence spectroscopy in renal ischemia and reperfusion: noninvasive evaluation of organ viability," Transplant. Proc. 45(5), 1715-1719 (2013).

38. E. O. Olweny and J. A. Cadeddu, Light Reflectance Spectroscopy and Autofluorescence (Kidney and Prostate), Springer, New York (2015).

39. R. N. Raman et al., "Optical spectroscopy approach for the predictive assessment of kidney functional recovery following ischemic injury," Proc. SPIE 7561, 756109 (2010).

40. J. T. Fitzgerald et al., "Real-time assessment of in vivo renal ischemia using laser autofluorescence imaging," J. Biomed. Opt. 10(4), 044018 (2005)

41. L. F. Tirapelli et al., "Renal ischemia in rats: mitochondria function and laser autofluorescence," Transplant. Proc. 40(5), 1679-1684 (2008).

42. R. N. Raman et al., "Quantification of in vivo autofluorescence dynamics during renal ischemia and reperfusion under $355 \mathrm{~nm}$ excitation," Opt. Express 16(7), 4930-4944 (2008).

43. R. N. Raman et al., "A non-contact method and instrumentation to monitor renal ischemia and reperfusion with optical spectroscopy," Opt. Express 17(2), 894-905 (2009).

44. L. F. Tirapelli et al., "Histopathology and laser autofluorescence of ischemic kidneys of rats," Lasers Med. Sci. 24(3), 397-404 (2009).

45. R. N. Raman et al., "Evaluation of the contribution of the renal capsule and cortex to kidney autofluorescence intensity under ultraviolet excitation," J. Biomed. Opt. 14(2), 020505 (2009).

46. P. E. Debevec and J. Malik, "Recovering high dynamic range radiance maps from photographs," in Proc. of the 24th Annual Conf. on Computer Graphics and Interactive Techniques, Los Angeles, pp. 369-378, ACM Press/Addison-Wesley Publishing Co. (1997).

47. E. Reinhard et al., High Dynamic Range Imaging: Acquisition, Display, and Image-Based Lighting, 2nd ed., Morgan Kaufmann, Burlington, Massachusetts (2010)

48. R. K. Chaurasiya and K. R. Ramakrishnan, "High dynamic range imaging," in Proc. of the 2013 Int. Conf. on Communication Systems and Network Technologies, pp. 83-89, IEEE Computer Society (2013).

49. P. Fei et al., "High dynamic range optical projection tomography (HDROPT)," Opt. Express 20(8), 8824-8836 (2012).

50. C. Vinegoni et al., "Real-time high dynamic range laser scanning microscopy," Nat. Commun. 7(1), 11077 (2016).

51. L. Lian et al., "High-dynamic-range fluorescence molecular tomography for imaging of fluorescent targets with large concentration differences," Opt. Express 24(17), 19920-19933 (2016).

52. Q. Tang et al., "High-dynamic-range fluorescence laminar optical tomography (HDR-FLOT)," Biomed. Opt. Express 8(4), 2124-2137 (2017).

53. F. Banterle et al., Advanced High Dynamic Range Imaging: Theory and Practice, AK Peters (CRC Press), Natick, Massachusetts (2011).

54. R. Fattal, D. Lischinski, and M. Werman, "Gradient domain high dynamic range compression," ACM Trans. Graph. 21(3), 249-256 (2002).

55. Y. Li et al., "Primed 3D injectable microniches enabling low-dosage cell therapy for critical limb ischemia," Proc. Natl. Acad. Sci. U. S. A 111(37), 13511-13516 (2014)

56. A. V. DSouza et al., "Logarithmic intensity compression in fluorescence guided surgery applications," J. Biomed. Opt. 20, 80504 (2015).

57. R. C. Gonzalez, R. E. Woods, and S. L. Eddins, Digital Image Processing Using MATLAB, Prentice Hall, Upper Saddle River, New Jersey (2003).

58. H. K. Eltzschig and T. Eckle, "Ischemia and reperfusion-from mechanism to translation," Nat. Med. 17, 1391-1401 (2011).

59. M. Malek and M. Nematbakhsh, "Renal ischemia/reperfusion injury; from pathophysiology to treatment," J. Renal Inj. Prev. 4(2), 20-27 (2015). 
60. P. P. Kapitsinou and V. H. Haase, "Molecular mechanisms of ischemic preconditioning in the kidney," Am. J. Physiol. Renal Physiol. 309(10), F821-F834 (2015).

61. S. Kumar, "Cellular and molecular pathways of renal repair after acute kidney injury," Kidney Int. 93(1), 27-40 (2018).

Yang Gao received his bachelor's degree in pharmaceutical sciences from Tsinghua University, Beijing, China, in 2014. He is currently a $\mathrm{PhD}$ candidate in the Department of Biomedical Engineering, Tsinghua University, Beijing, China. His research interest is dynamic fluorescence molecular imaging for small animal imaging.

Yuan Zhou received his bachelor's degree in biomedical engineering from Beijing Jiaotong University, Beijing, China, in 2014. He is currently a PhD candidate in the Department of Biomedical Engineering, Tsinghua University, Beijing, China. His research interest is fluorescence molecular tomography for small animal imaging.
Fei Liu is an associate professor at Beijing Jiaotong University. She received her $\mathrm{PhD}$ in biomedical engineering from Tsinghua University in 2013 and was a postdoctoral research scientist from 2013 to 2015 in School of Medicine at Tsinghua University. Her research interest is fluorescence molecular tomography for small animal imaging. She has authored or coauthored over 60 journal papers.

Jianwen Luo is a professor at Tsinghua University. He was enrolled in the Thousand Young Talents Program in 2012, received the excellent young scientists fund from the National Natural Science Foundation of China in 2013, and was supported by the Young Scientists Project of National Key R\&D Program of China in 2016. His research interests include ultrasound elasticity imaging and fluorescence molecular imaging. He has published more than 130 journal papers and 50 conference papers. 